\title{
Performance Evaluation of Emergency Messaging via Wireless Collision Avoidance Systems for Improved Traffic Safety in VANET
}

\author{
Prema G \\ Electronics and communication \\ Mepco Schlenk Engineering College \\ Sivakasi, India
}

\author{
Kalpana C \\ Electronics and communication \\ Mepco Schlenk Engineering College \\ Sivakasi, India
}

\begin{abstract}
Vehicles move on the roads sharing information between themselves and with the Internet through the access points. In this paper, a collision avoidance system for Vehicular Ad-hoc Networks is provided and a comparative study is done between without $\mathrm{CA}$ system and with $\mathrm{CA}$ system for vehicular safety critical application. In a Vehicular Ad Hoc Network (VANET), the wireless Collision Avoidance (CA) system issues warnings to drivers before they reach a potentially dangerous zone on the road. CA system immediately generates an emergency warning message to vehicles behind, before they reach the accident zone. There by improving road safety and travel comfort by enabling the inter-vehicular communications. Various metrics like packet delay, packet delivery fraction, overhead are used for evaluating the performance of emergency messaging via wireless CA system.
\end{abstract}

\section{Keywords}

Wireless Collision Avoidance system, VANET, Dedicated short range communication. Rear end collision

\section{INTRODUCTION}

VANETs are used for short distance and the communication is high speed among nearby vehicles or vehicle to vehicle or between vehicles and roadside infrastructure units [1]. Diverse wireless technologies are utilized for sharing data and providing data services to Vehicular Ad hoc Network. Vehicles communicate with each other through Dedicated Short Range communication (DSRC) wireless devices.

A typical VANET consists of vehicles and access points along the road. Vehicles move on the road sharing information between them and with the Internet through the access points. Vehicles often move at high speed but their mobility is rather regular and predictable [2]. High speed movement creates scenarios characterized by a very dynamic network topology. Vehicles can always rely on recharging batteries. An accurate estimate of vehicles position can be made available through GPS systems or on-board sensors.

Vehicle-to-Vehicle (V2V) communication supports services such as car collision avoidance and road safety by exchanging warning messages across vehicles [3]. An integration of VANET and 3G networks using mobile gateways (i.e., vehicles) are introduced. The envisioned architecture shall enable mobile data access for vehicles, anytime and anywhere. In particular, the integration of IEEE 802.11-based multi-hop VANETs with $3 \mathrm{G}$ shall contribute to the evolution of beyond $3 \mathrm{G}$ (B3G) wireless communication systems. As an integral part of the architecture, UMTS enables mobile data access to vehicles, offering a wide range of communication of around 8 to $10 \mathrm{~km}$ per BST.

The VSC (Vehicle Safety Communications) project identifies vehicle safety applications enabled by DSRC (Dedicated short range communication) communications [7], [8]. The applications for traffic safety have been widely developed. In existing system vehicle cooperative collision avoidance application is based on dedicated short range communication. Chain collision can be severity lessened by reducing the delay between the time of an emergency event and the time at which the vehicles behind are informed about it[9].MAC performance of a periodic safety message mechanism in terms of the reception probability and the channel busy time[10]. In sparse VANET, two vehicles are probably disconnected and the message delivery is taken by the store-carry forward scheme[11]-[13]. In such a case, a driver can recognize the hazard as fast as possible. Therefore, the succeeding vehicles have enough time to make a safety stop, while vehicles further away can also quickly change lane or make reroute decisions accordingly. . A scheme as per [14] allows vehicle to estimate the local density and distinguish between the free flow and the congested traffic phases. Based on the local traffic conditions, a vehicle can set a suitable transmission range dynamically according to the traffic density [15]

\section{PROPOSED METHOD}

The proposed system shows how vehicle-to-vehicle communications can enhance highway traffic safety with minimum wireless latency so that the driver has more available maneuvering time to react to hazardous situations as in Fig 1, and also the proposed system has high packet delivery fraction and low routing overhead compared to already existing method of DSRC. The proposed method also demonstrates the need for data prioritization in VANET, as high data priority is given to UMTS based vehicle like ambulance, compared to ordinary vehicle by the CA base station for safety critical applications. This is an additional feature implemented along with CA system in the proposed work. So combining both wireless collision avoidance systems along with data prioritization, into a single VANET model reduces number of car crashes as the probability of vehicles failing to receive the emergency message is very much negligible because the wireless propagation delay is significantly smaller than the cumulative driver's reaction time, since a driver who receives an early warning can have more time to react in an emergency situation. A comparative 
study is made based on rear end collision avoidance with CA system having CA base station as ad-hoc relay and rear collision occurrence without CA system using RSU as ad-hoc relay and the efficacy of the proposed framework has been analyzed and validated by extensive simulation results.

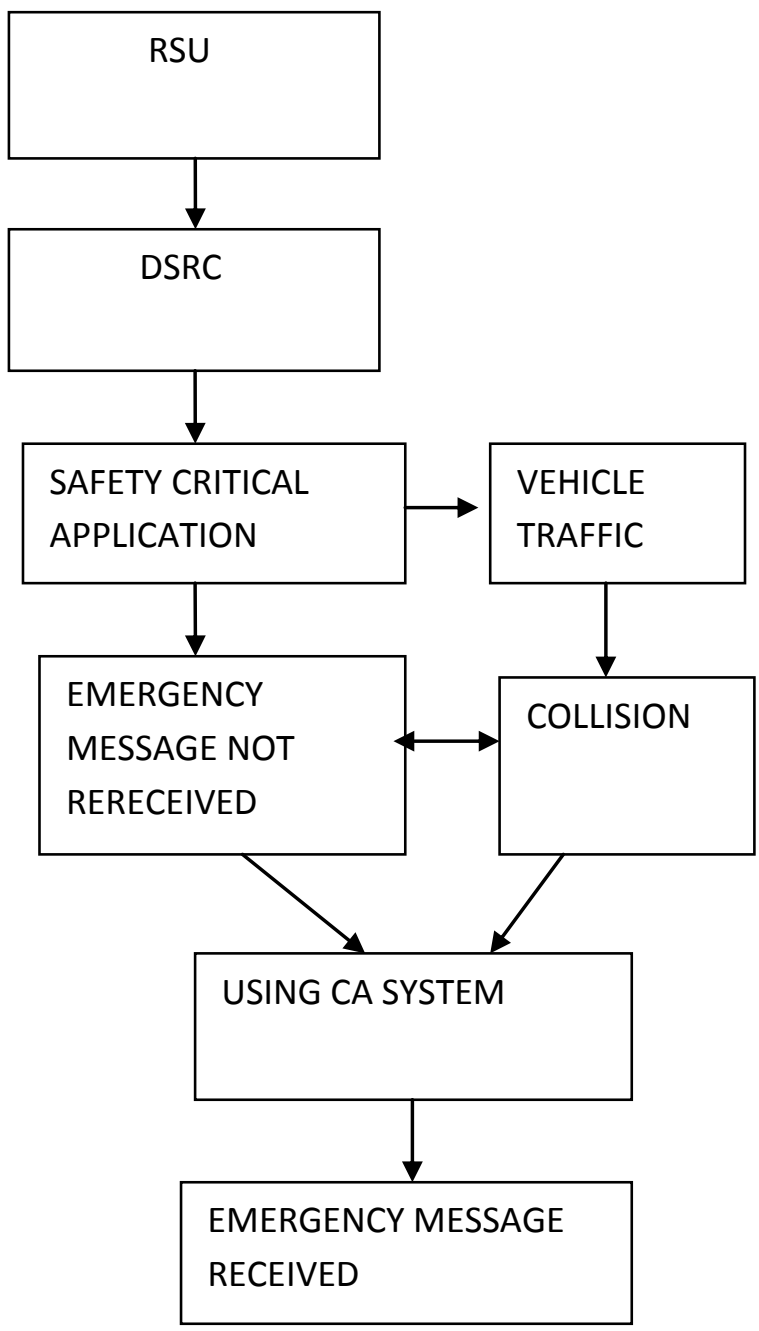

Fig 1: Block diagram of proposed method

\section{COMPARATIVE STUDY OF CA AND WITHOUT CA SYSTEM FOR REAR END COLLISION}

A specific, on-board wireless Collision Avoidance (CA) system is used to warn drivers before they reach a potentially dangerous zone on the road. When a sudden event occurs on the dangerous road, accident occurs and to avoid the accident or a vehicular crash, emergency braking is applied. A vehicle equipped with the wireless $\mathrm{CA}$ system can immediately generate an emergency message to vehicles behind it. So to avoid the accident and avoid the collision had to go for $\mathrm{v} 2 \mathrm{v}$ data communication using collision avoidance systems. To properly design a wireless CA system, it is essential to evaluate whether vehicles located in the potentially dangerous zone are connected to VANET. When vehicles are connected to VANET, the drivers can immediately receive emergency messages. In such cases, drivers have more time to react to hazards.
Driving means changing location which means there is a constant demand for information on the current location and specifically for data on the surrounding traffic, routes and much more. There are various groups of information like assisting the driver in car safety. There are various things based on sensor information from various vehicles like brake warning sent from preceding car, collision warning, information about road condition, weather forecast of the region, premonition of traffic jams, and caution for any accident in the next turn in the lane, and information to be given for the team which arrives to rescue. Updates on the navigation systems of car is also provided

\subsection{Rear end collision occurrence without CA system}

A practical VANET model is developed to compute the probability of a rear-end collision without a wireless CA system. A rear-end collision between two vehicles traveling in the same direction occurs when a sudden braking situation occurs. In the V2V, closely moving front vehicle suddenly puts on the brake, so vehicle crash or accident may occur. Delay of the emergency message occurs when not using wireless collision avoidance system. Using normal road side unit network, emergency message is received but delay is involved to deliver to vehicles in existing method. The headway denotes the distance between the front of one vehicle and the front of the subsequent vehicle.

Rear end collision occurs when there is stringent delay as available maneuvering time is less than needed maneuvering time. Needed maneuvering time depends on driver's perception response time. To prevent rear end collision vehicle must receive message prior to front vehicle's deceleration to provide more available maneuvering time.

\subsection{Rear-end collision avoidance with $\mathrm{CA}$ System}

Rear end collision is avoided using a CA base station. First it improves the network connectivity. Second it increases message delivery options in both V2 V and V2I aspects. Third it reduces the delivery delay or sending a safety message to disconnected vehicles. In wireless CA system even though some further vehicles are not located in the dangerous zone, these vehicles can still utilize the immediate warning for route planning. The CA failure rate is to indicate the probability that at least one disconnected vehicle located in the ROI failing to receive the emergency message. This output metric can be served as an important metric to determine the number of RSUs needed to be deployed in future intelligent transportation.

Why emergency message is not received: A rear-end collision between two vehicles that travel in the same direction that do not using CA system does not receive emergency message. So, using wireless collision avoidance system before meeting the dangerous zone, the vehicle gets the emergency message, for example braking, lane changes, and acceleration, vehicle to vehicle distance etc.

Wireless Collision Avoidance System: The CA system must keep broadcasting the latest road information more frequently to drivers when the detection of traffic density by the VANET safety application is within this critical range. A vehicle equipped with the wireless CA system can immediately generate an emergency message to vehicles behind it. So to avoid the accident and avoid the collision, go for $\mathrm{v} 2 \mathrm{v}$ data communication using collision avoidance systems 
Advantage for wireless Collision Avoidance System: The advantage of proposed system considerably improves road safety and travel comfort by enabling inter-vehicle communications. With a wireless CA system, the damage after an accident would be not much serious, thereby improving traffic safety.

\section{RESULTS AND DISCUSSION}

\subsection{Without CA system implementation}

A practical VANET model is implemented with 10 vehicular nodes, of which 2 nodes are configured as static RSU and 8 nodes as dynamic vehicular nodes. The existing system of DSRC is implemented and rear end collision between vehicles is simulated using NS 2.34.Collision scenario is shown as visual demonstration in Nam output in Fig. 2 and 3 as emergency messages are either not received or received with delay.

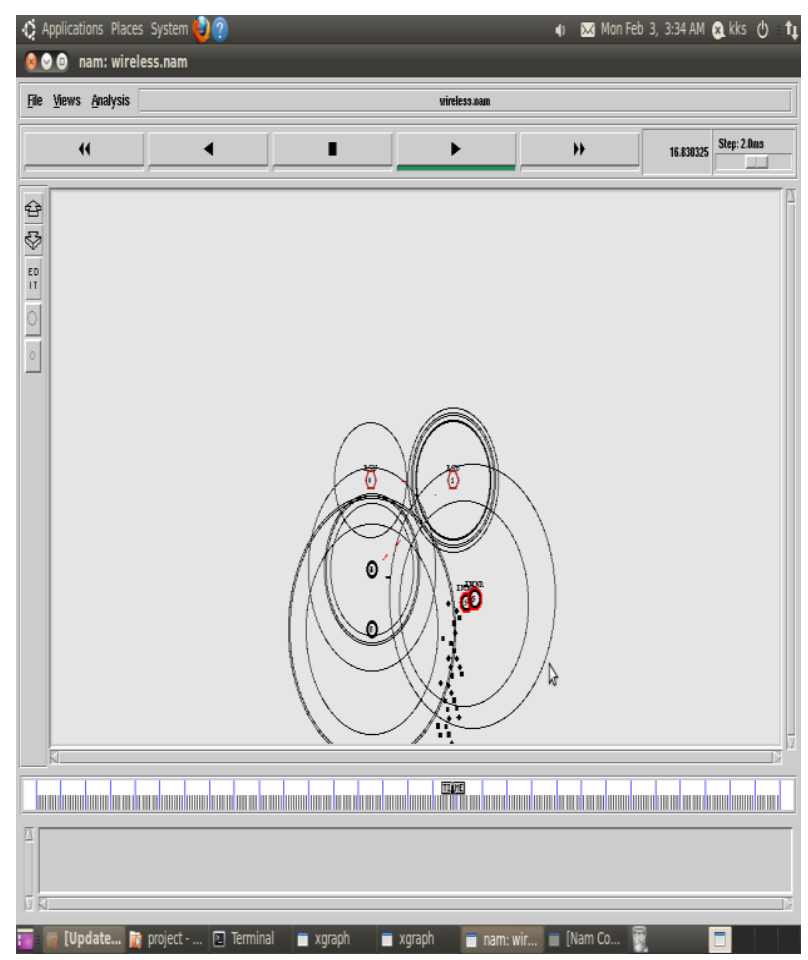

Fig 2: Nam output of Rear end collision without CA system

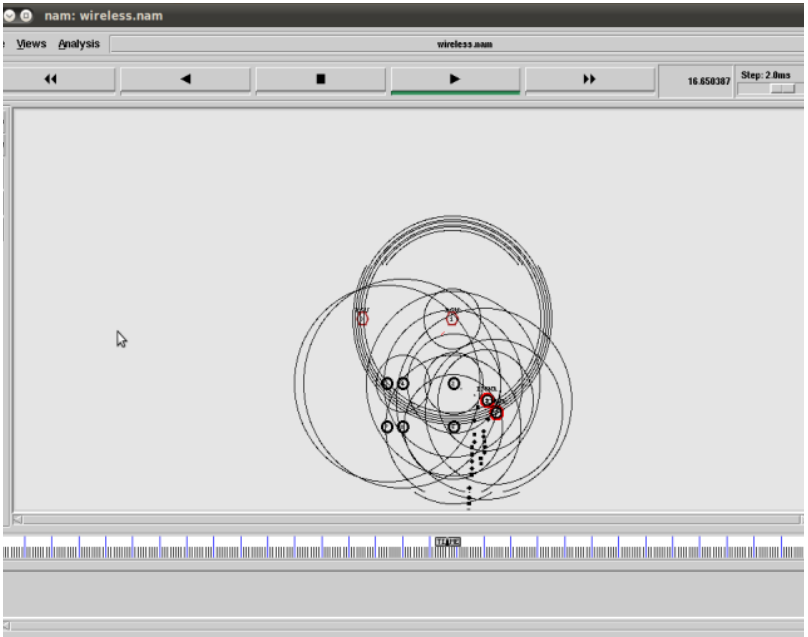

Fig: 3 Nam output showing dropped packets in non CA system

For the above said VANET scenario, $\mathrm{X}$ graph for packet loss is obtained in Fig.5 as event driven packets that contains emergency messages are not reaching vehicular nodes as a result of which, there results collision.

\subsection{With CA system implementation}

A practical VANET model with CA base station is implemented in Fig.4 with 10 vehicular nodes, of which 4 are wired nodes, other nodes are wireless. UMTS based vehicle is represented as blue color and ordinary vehicles as black. High priority packets are represented as blue colored packets, thus data prioritization and collision avoidance is combined in CA System in Fig.4.

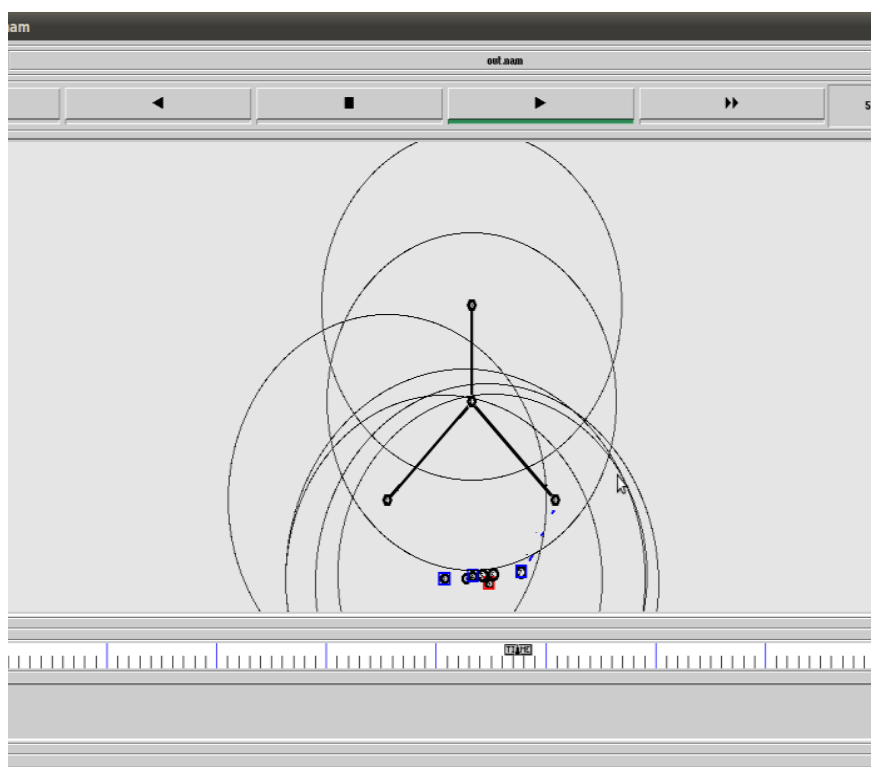

Fig 4: Nam output of CA system with data prioritization 


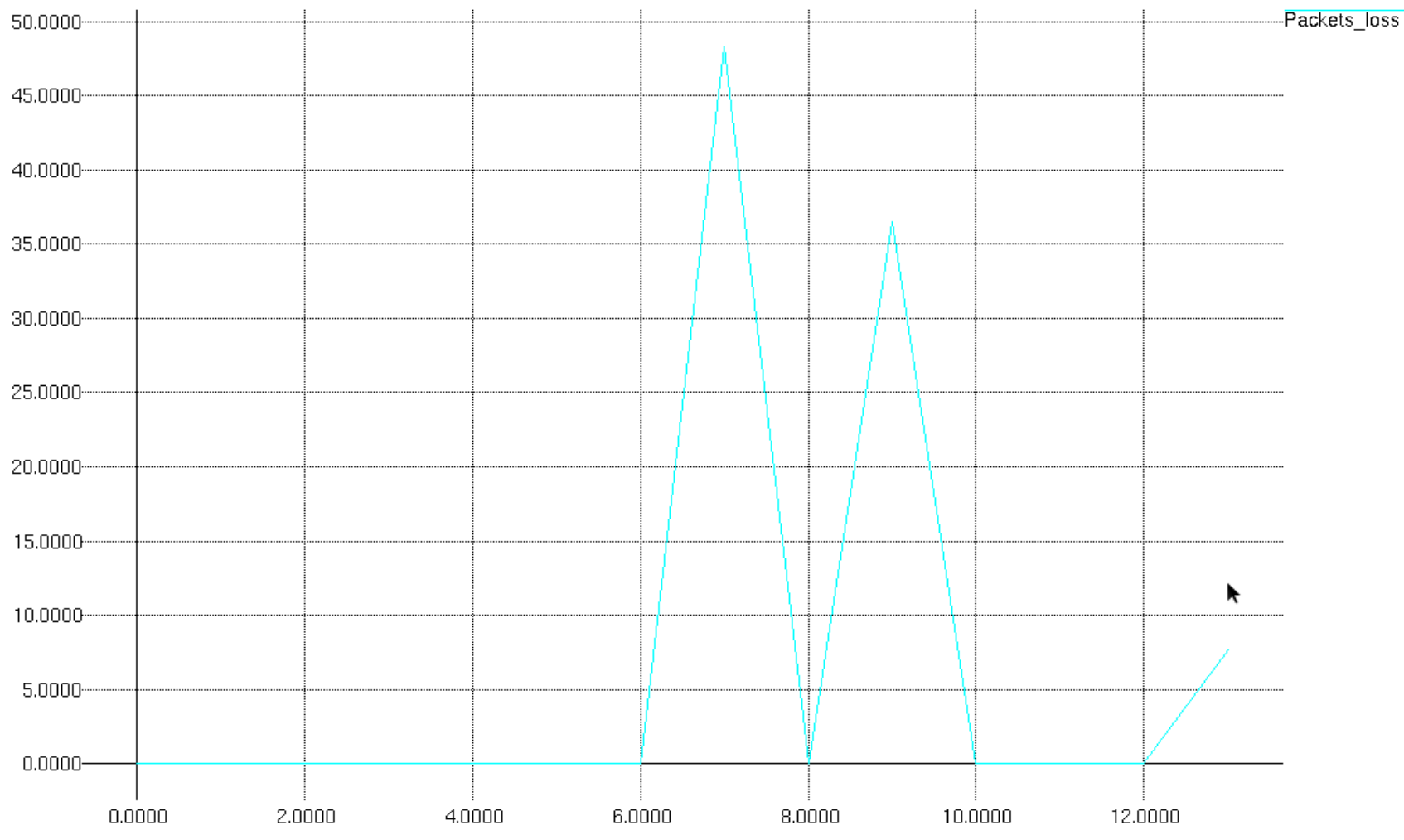

Fig 5: Plot of packet loss without CA system

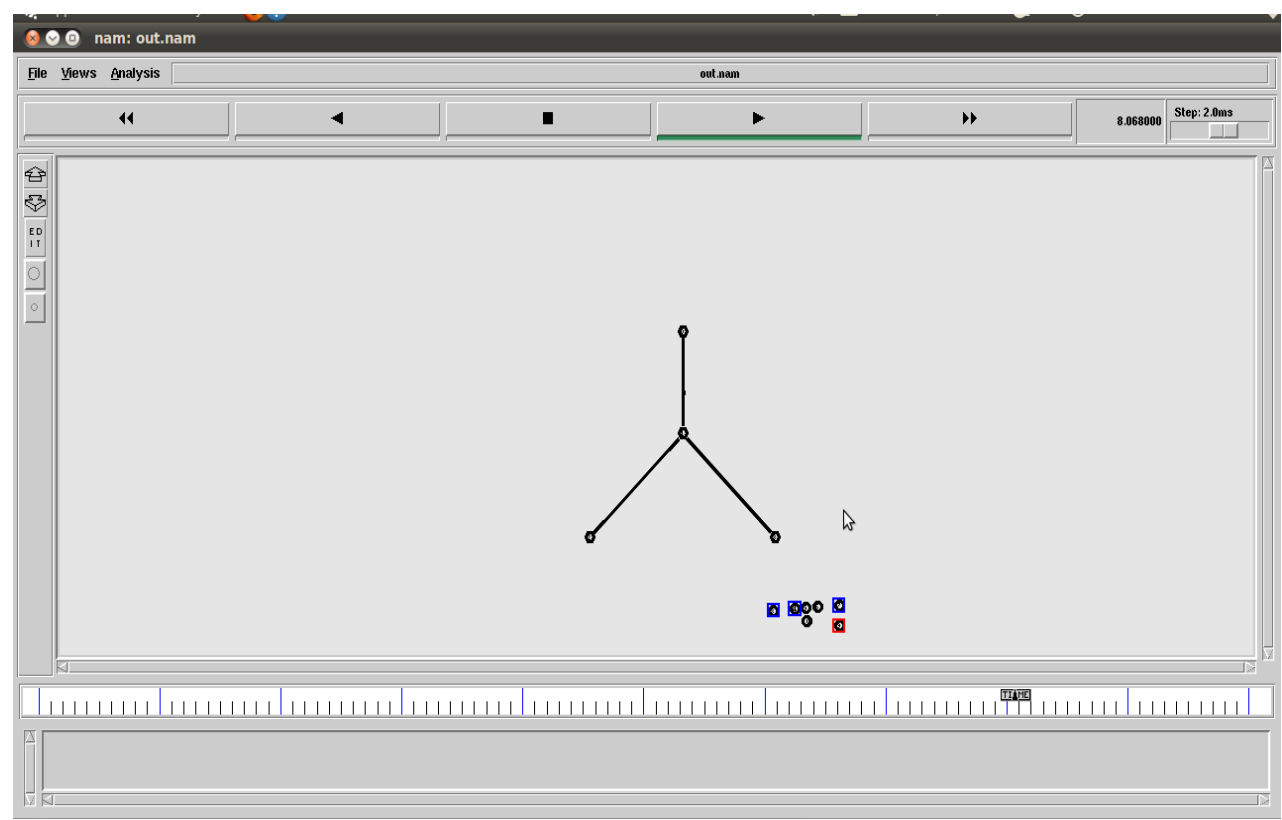

Fig 6: Nam output of CA system

Thus in spite of congested phase of traffic, UMTS vehicles had made their way through avoiding collision with ordinary vehicles as high data prioritization is combined here with CA system in Fig 6. The plot of collision avoidance system with normal and high priority is seen in Fig.7

There is high successful packet delivery for CA system compared to that of without CA system, thereby emergency messages are received without getting dropped in CA system with high success rate compared to without CA system. It is seen there is low overhead for high priority, CA based system compared to that of without CA based system in Fig.8, as we know high priority means low overhead. 
Vo of Packets(\%)
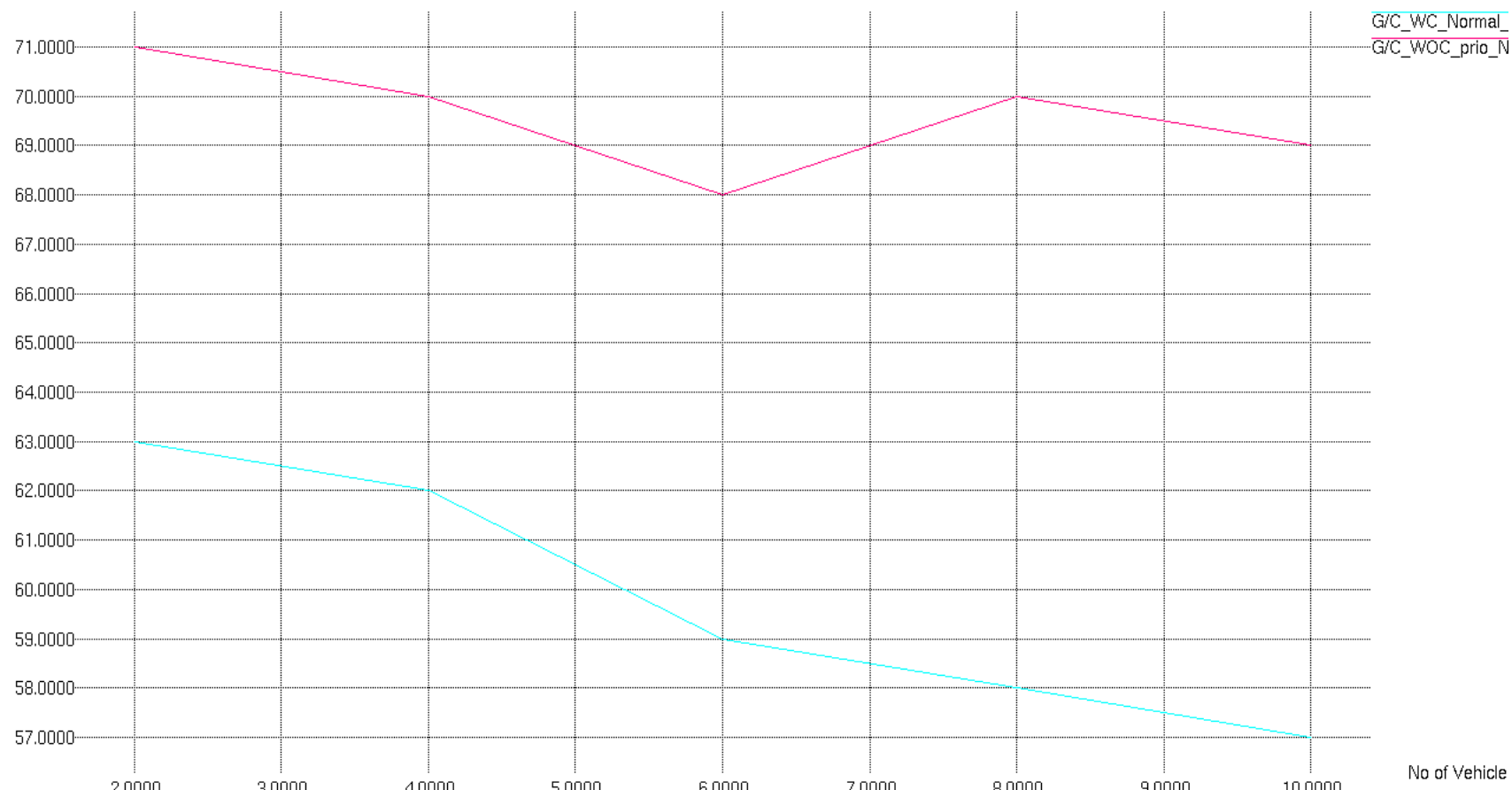

Fig7: Plot of Comparison of CA and without CA system in VANET for safety critical application

Liose LHacpy Anour

$\mathrm{PKT}(\mathrm{Kb})$

76.0000

74.0000

72.0000

70.0000

68.0000

66.0000

64.0000

62.0000

60.0000

58.0000

56.0000

54.0000

52.0000

50.0000

48.0000

46.0000

44.0000

42.0000

40.0000

38.0000

36.0000

34.0000

(1)

$\mathrm{OH}$

X/\{OH\}_Normal_N $\bar{X}\{\mathrm{OH}\}+\mathrm{H} \_$prio_N

Fig: 8: Plot of comparison of overhead with $\mathrm{CA}$ and without CA system 


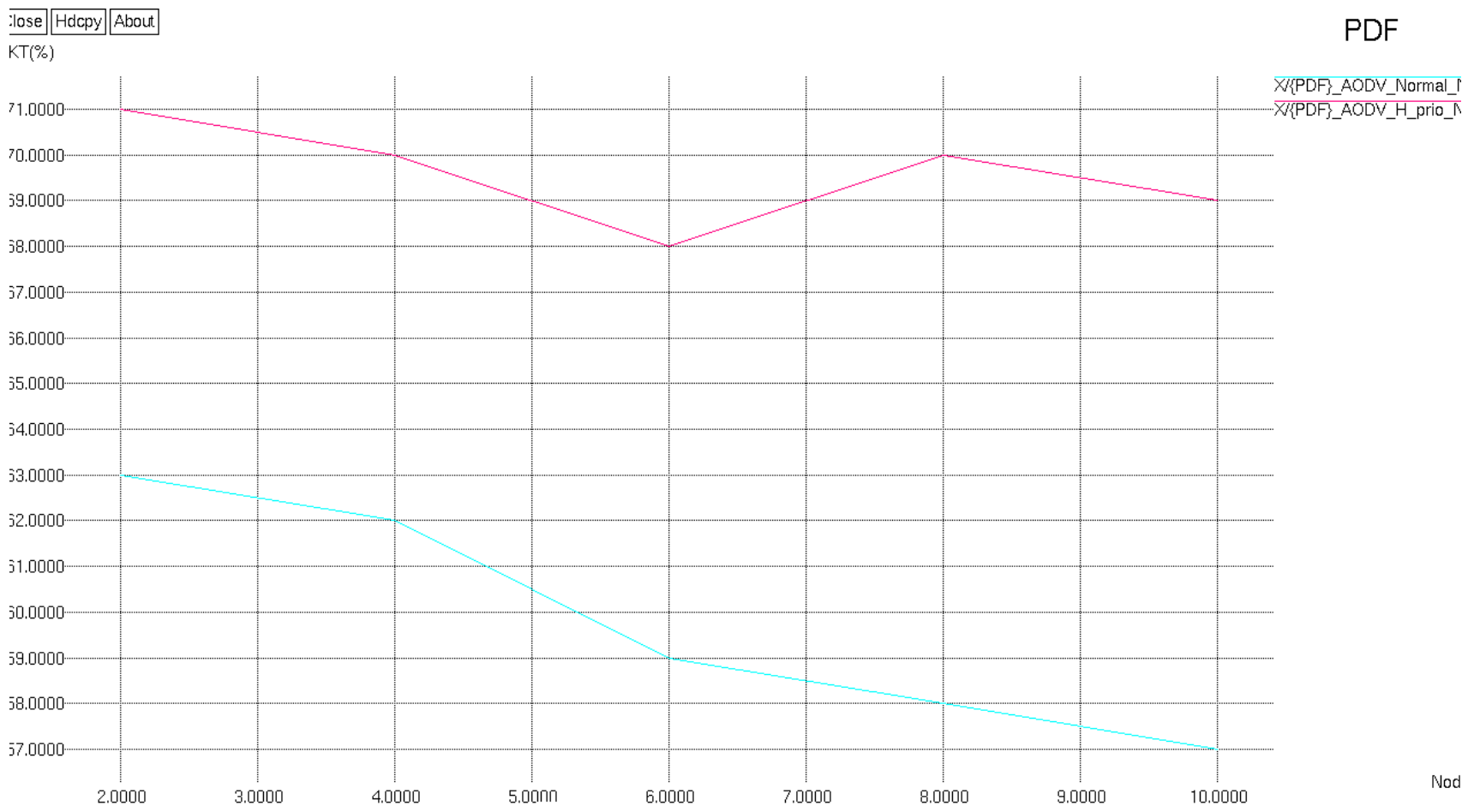

Fig.9: Plot of comparison of Packet delivery fraction with CA and without CA system

It is seen that there is high Packet delivery fraction or throughput for CA based system compared to without CA system as can be seen in Fig.9.

\section{CONCLUSION}

Among a vast array of potential applications, emergency messaging has attracted much attention in the literature. When vehicles are connected to VANET, drivers can immediately receive emergency messages via direct transmission. In such instances, drivers have enough time to react to hazardous situations appropriately. For instance, vehicles near the accident site can slow down or stop before colliding with the preceding vehicle, while vehicles further away can quickly change their lanes or make detour/reroute decisions accordingly. The performance of a wireless collision avoidance system with or without employing RSUs as ad-hoc relays and the efficacy of the proposed framework has been analyzed and validated by extensive simulation results. The simulation results indicated that the vehicle density in a critical range is more prone to chain collisions. The CA system must keep broadcasting the latest road information more frequently to drivers when the detection of traffic density by the VANET safety application is within this critical range.

\section{REFERENCES}

[1] F.Bai,T.Elbatt,G.Hollan,H.Krishnan and V.Sadekar "Towards characterizing and classifying communication-based automotive applicationsfrom a wireless networking perspective", in Proc, 2006 IEEE workshop Automotive new

[2] S. Biswas, R. Tatchikou, and F. Dion, "Vehicle-tovehicle wirele communication protocols for enhancing highway traffic safety," IEEE Commun. Mag., vol. 44, no. 1, pp. 74-82, Jan. 2006

[3] Y.Zhang,E.K.Antonosson and K.Grote, "A new threat assessment measure for collision avoidance systems," in Proc.2006 IEEE Intelligent Transportation Syst.Conf.pp.968-975,sept.2006

[4] J.R. McLean,Two lane Highway traffic Operations:Theory and Practice.Gordon and Breach Science publishers, 1989

[5] N.Gartner, C.J.Messer and A.K.Rathi, "Revised monograph on traffic flow theory,"Transportation Research Board,1999

[6] H.Greenberg, "An anlysiof analysiss of traffic flow,"Operations Research,vol 7,no.1,pp,7985,jan/feb 1959

[7] NHTSA, "Second Annual report of the crash avoidance metrics partnership,april 2002-march 2003" in national highway traffic safety administration,DOT HS 809 663,Jan 2003

[8] NHTSA "Third Annual report of the crash Avoidance metrics partnership,April 2003-march 2004," in National highway traffic safety administration,DOT HS 809 837,feb 2005

[9] Q.Xu.RSengupta and D.Jiang,"design and analysis of highway safety communication protocol in $5.9 \mathrm{GHz}$ dedicated short range communication Spectrum," IEEE Trans .Veh.Tech vol 57 no 4 pp 2451-24555, 2003

[10] Xu.T.Mak.J.Ko and Sengupta,"vehicle to vehicle safety messaging in DSRC in PROC 2004 ACM VANET 
[11] J.Yin,et al,"Performance analysis of safety applications over DSRC vehicular adhoc network",in Proc 2004 ACM international workshop veh adhoc netw,pages 1-9

[12] X.Yang,J.Liu, F.Zhao and N.H.Vaidya,"A vehicle to vehicle communication protocol for cooperative collision warning", in Proc 2004 International conf mobile ubiquitous system Netw Services,pp114-123

[13] C.L.Huang,Y.P.Fallah,R.Sengupta and H.Krishnan,"Adaptive intervehicle communication control for cooperative safety systems",IEEE netw Mag.,vol.24,no.1,pp6-13,jan/feb 2010.

[14] M.Artimy,'KLocal density estimation and dynamic transmission range assignment in vehicular ad-hoc network",IEEE Trans.Intelligent Transportation System vol 8 no.3,pp400-412,sept2007

[15] M.Torrent-Moreno,J.Mittang,P.Santi and H.Hartenstein,"Vehicle to vehicle communication:Fair transmit power control for safety critical information",IEEE Trans Veh Tech vol 58 no 7 pp 3684-3703,sept 2009 